\title{
First Report of Temnocephala brevicornis Monticelli 1889 (Temnocephalidae: Platyhelminthes) in Argentina
}

\author{
Francisco Brusa ${ }^{+}$, María Cristina Damborenea
}

\author{
Departamento Científico Zoología Invertebrados, Facultad de Ciencias Naturales y Museo, UNLP, Paseo del \\ Bosque s/no, 1900 La Plata, Argentina
}

Temnocephala brevicornis Monticelli 1889, ectosymbiont of Hydromedusa tectifera Cope 1869, is reported for the first time for Argentina. Numerous temnocephalans from Arroyo Villoldo in the locality of Magdalena, Buenos Aires, Argentina were stained in toto to be studied. This commensal species in turtles was originally cited in association with Hydromedusa maximiliani (Mikan) and Hydraspis radiolata Mikan in Brazil. Afterwards, it was found on other fresh water turtle species in Brazil and Uruguay.

Key words: Temnocephala - Hydromedusa tectifera - South America - Argentina

The Temnocephalidae includes symbiotic turbellarians from Australia, New Zealand, New Guinea, Madagascar, Central and South America (Williams 1981). The first temnocephalan described was Temnocephala chilensis (MoquinTandon, 1846) found in association with an anomuran crab, Aegla laevis (Laitreille, 1818), from near Santiago, Chile. Since then, 19 species of Temnocephala have been reported in South and Central America associated with a large variety of hosts (crustaceans, molluscs, insects and turtles). Nine of these have been found in Argentina (Damborenea 1992).

The present short communication represents the first record of T. brevicornis for Argentina. This species was found on the surface of Hydromedusa tectifera Cope 1869 . This turtle has a wide distribution in South America; from the states of Rio Grande do Sul and Santa Catarina in Brazil, Paraguay and Uruguay. In Argentina $H$. tectifera can be found from the northeast (Formosa, Chaco, Santa Fé, Córdoba, Misiones, Corrientes and Entre Ríos) to the latitude of $37^{\circ}$ South in the basin of the Salado river, Buenos Aires (de la Fuente 1992)

T. brevicornis Monticelli, 1889 is the only species to have been found on a turtle. It was first described associated with H. maximiliani (Mikan) and Hydraspis radiolata Mikan in Brazil. Subsequently,

\footnotetext{
Supported by CIC and Conicet.

+Corresponding author. Fax: 54221 4257527. E-mail: franbrusa@yahoo.com

Received 15 July 1999

Accepted 11 November 1999
}

other hosts and localities have been reported, as follows:

H. maximiliani (Mikan) from Teresópolis, Rezende, Angra dos Reis, Rio de Janeiro, and Viçosa, Minas Gerais, Brazil (Pereira \& Cuocolo 1940, 1941);

H. tectifera Cope from São Paulo, Brazil (Pereira \& Cuocolo 1941); Valle Edén, Tacuarembó, Uruguay (Dioni 1967);

Mesoclemmys (Schw.) (=Hydraspis Schw.) Brazil (Monticelli 1899);

Acanthochelys spixii (Duméril et Bibron, 1835) Viamão, Rio Grande do Sul, Brazil (Ferreira Yuki et al. 1993);

Trachemys dorbigni (Duméril et Bibron, 1835) Viamão, Rio Grande do Sul, Brazil (Ferreira Yuki et al. 1993).

Thus, the presence of T. brevicornis is known from Brazil and Uruguay. Moreover it has also been refereed in association to Pseudotelphusa sp. (Crustacea) in Venezuela and in El Salvador (Lamothe Argumedo 1974). However, this reference is in some doubt since it is the only one for a crustacean host.

Numerous temnocephalans were found on the surface of three specimens of $H$. tectifera, from Arroyo Villoldo and Ruta 11, in the locality of Magdalena, Buenos Aires, Argentina $\left(35^{\circ}\right.$ $\left.15.936^{\prime} \mathrm{S}-57^{\circ} 15.5366^{\prime} \mathrm{W}\right)$. Owing to their large size and active movements, these commensals are very evident when they alive. Approximately 50 young and adult specimens were fixed in $10 \%$ formaldehyde, stained with hydrochloric carmine and mounted in Canadian balsam. The specimens have been deposited in the Helminthological Collection of the La Plata Museum (MLP 4585) Argentina. 
TABLE

Temnocephala brevicornis Monticelli 1889. Specimen measurements from Arroyo Villoldo Argentina. The measurements given by Pereira and Cuocolo (1940) are shown in parenthesis

\begin{tabular}{lccc}
\hline Measurements & $\overline{\mathrm{X}}$ & $\mathrm{N}$ & $\mathrm{DS}$ \\
\hline Total length $(\mathrm{mm})$ & $2.439(3.70)$ & $27(10)$ & $0.551(0.806)$ \\
Maximum width $(\mathrm{mm})$ & $1.544(2.17)$ & $27(10)$ & $0.3959(0.358)$ \\
Sucker diameter $(\mu \mathrm{m})$ & $653.28(950)$ & $26(10)$ & $126.08(114)$ \\
Pharynx $(\mu \mathrm{m})$ & & & \\
Width & $410.74(650)$ & $16(6)$ & $95.874(107)$ \\
Length & $386.24(670)$ & $16(6)$ & $87.008(114)$ \\
Penis $(\mu \mathrm{m})$ & & & 29.7 \\
Total length & $291.3(360)^{a}$ & 27 & 1.17 \\
Basal width & $52.19(100)^{a}$ & 27 & 2.056 \\
Distal width & $11.84(40)^{a}$ & 26 & 105 \\
Spine zone length & 28.5 & 26 & 15.8 \\
Seminal vesicle $(\mu \mathrm{m})$ & & & 29.88 \\
Width & $66.67(80)^{a}$ & 11 & \\
Length & $200.1(340)^{a}$ & 11 & 22.308 \\
Penis bulb $(\mu \mathrm{m})$ & & & 33.25 \\
Width & $73.44(120)^{a}$ & 16 & \\
Length & $82.54(290)^{a}$ & 16 & \\
Total length/sucker diameter $(\mu \mathrm{m})$ & $3.7335(3.89)^{a}$ & & \\
Total length/penis length $(\mu \mathrm{m})$ & $8.3728(10.27)^{a}$ & & \\
Penis length/bulb length $(\mu \mathrm{m})$ & $3.5292(4.39)^{a}$ & & \\
Penis length/vesicle length $(\mu \mathrm{m})$ & $1.4558(1.058)^{a}$ & & \\
\hline & & &
\end{tabular}

$a$ : Pereira and Cuocolo (1940) only mention these measurements.

T. brevicornis has been well described by Pereira and Cuocolo (1940). These authors described the penis, now considered most relevant in specific identification (Cannon 1993), as a conical elongated structure $360 \mu \mathrm{m}$ long, $100 \mu \mathrm{m}$ of basal diameter and $40 \mu \mathrm{m}$ of distal diameter. The distal end is slightly dilated. The inner distal surface has several rows of small thorns. These features and measurements have also been reported by Ferreira Yuki et al. (1993).

The specimens studied here presented similar structural characteristics to those studied by Pereira and Cuocolo (1940), but were smaller (Table).

In particular the penis is shorter and narrower but it demonstrates the same morphology.

\section{REFERENCES}

Cannon LRG 1993. New temnocephalans (Platyhelminthes): ectosymbionts of freshwater crabs and shrimps. Men Queensland Museum 33: 17-40.

Damborenea MC 1992. Especies de Temnocephala (Platyhelminthes, Temnocephalidea) de crustaceos y moluscos de Argentina. Iheringia Sér Zool 72: 3-23.

de la Fuente MS 1992. Las tortugas Chelidae del Terciario superior y Cuaternario del territorio argentino. Ameghiniana 29(3): 211-229.
Dioni W 1967. Temnocephalas uruguayas II. Descripción de Temnocephala talicei $\mathrm{n}$. sp. y notas sobre T. axenos Monticelli (Platyhelmintha). Physis 26: 477-484.

Ferreira Yuki VL, Damborenea MC, Osorio Mallman MT 1993. Acantochelys spixii (Duméril et Bibron, 1835) (Chelidae) e Trachemys dorbigni (Duméril et Bibron, 1835) (Emydidae) (Testudines) como hospedeiros de Temnocephala brevicornis Monticelli 1889 (Temnocephalidae) (Platyhelminthes). Comun Mus Ciênc PUCRS sér zool 6: 75-83.

Lamothe Argumedo R 1974. Algunas consideraciones sobre el género Temnocephala Blanchard, 1849, y descripción de una especie nueva de Costa Rica. An Inst Biol Univ Nac Aut México ser zool 45: 31-38.

Montcelli FS 1899. Sulla Temnocephala brevicornis Mont. 1889 e sulle temnocephale en generale. Bol Soc Nat Napoli 12: 72-127.

Pereira C, Cuocolo R 1940. Contribução para o conhecimento da morfología, bionomia e ecologia de "Temnocephala brevicornis Monticelli, 1889". Arq Inst Biol Brasil 11: 367-398.

Pereira C, Cuocolo R 1941. Estudos sobre "Temnocephalidae Monticelli, 1899", com estabelecimento de dois novos gêneros australianos e descrição de duas novas espécies neotrópicas. Arq Inst Biol Brasil 12: 101-127.

Williams JB 1981. Classification of the Temnocephaloidea (Platyhelminthes). J Nat Hist 15: 277-299. 